\title{
HS 2331+3905: The cataclysmic variable that has it all ${ }^{\star}$
}

\author{
S. Araujo-Betancor ${ }^{1, \star \star}$, B. T. Gänsicke ${ }^{2, \star \star}$, H.-J. Hagen ${ }^{3}$, T. R. Marsh ${ }^{2}$, \\ E. T. Harlaftis ${ }^{4}$, J. Thorstensen ${ }^{5}$, R. E. Fried ${ }^{6}$, P. Schmeer ${ }^{7}$, and D. Engels ${ }^{3}$ \\ ${ }^{1}$ Space Telescope Science Institute, 3700 San Martin Drive, Baltimore, MD 21218, USA \\ e-mail: araujo@stsci.edu \\ 2 Department of Physics, University of Warwick, Coventry CV4 7AL, UK \\ ${ }^{3}$ Hamburger Sternwarte, Universität Hamburg, Gojenbergsweg 112, 21029 Hamburg, Germany \\ ${ }^{4}$ Institute of Space Applications and Remote Sensing, National Observatory of Athens, PO Box 20048, Athens 11810, Greece \\ 5 Department of Physics and Astronomy, Dartmouth College, 6127 Wilder Laboratory, Hanover, NH 03755-3528, USA \\ ${ }^{6}$ Braeside Observatory, PO Box 906, Flagstaff AZ 86002, USA \\ 7 Bischmisheim, Am Probstbaum 10, 66132 Saarbrücken, Germany
}

Received 26 July 2004 / Accepted 27 September 2004

\begin{abstract}
We report detailed follow-up observations of the cataclysmic variable HS $2331+3905$, identified as an emissionline object in the Hamburg Quasar Survey. An orbital period of $81.08 \mathrm{~min}$ is unambiguously determined from the detection of eclipses in the light curves of HS $2331+3905$. A second photometric period is consistently detected at $P \simeq 83.38$ min, $\sim 2.8 \%$ longer than $P_{\text {orb }}$, which we tentatively relate to the presence of permanent superhumps. High time resolution photometry exhibits short-timescale variability on time scales of $\simeq 5-6 \mathrm{~min}$ which we interpret as non-radial white dwarf pulsations, as well as a coherent signal at 1.12 min, which is likely to be the white dwarf spin period. A large-amplitude quasi-sinusoidal radial velocity modulation of the Balmer and Helium lines with a period $\sim 3.5 \mathrm{~h}$ is persistently detected throughout three seasons of time-resolved spectroscopy. However, this spectroscopic period, which is in no way related to the orbital period, is not strictly coherent but drifts in period and/or phase on time scales of a few days. Modeling the far-ultraviolet to infrared spectral energy distribution of HS $2331+3905$, we determine a white dwarf temperature of $T_{\text {eff }} \simeq 10500 \mathrm{~K}$ (assuming $M_{\mathrm{wd}}=0.6 M_{\odot}$ ), close to the ZZCeti instability strip of single white dwarfs. The spectral model implies a distance of $d=90 \pm 15 \mathrm{pc}$, and a low value for the distance is supported by the large proper motion of the system, $\mu=0.14^{\prime \prime} \mathrm{yr}^{-1}$. The non-detection of molecular bands and the low $J, H$, and $K$ fluxes of HS 2331+3905 make this object a very likely candidate for a brown-dwarf donor.
\end{abstract}

Key words. stars: binaries: close - stars: individual: HS 2331+3905 - stars: novae, cataclysmic variables

\section{Introduction}

In the current standard model of cataclysmic variable (CV) evolution (see King 1988) the systems evolve to shorter orbital periods, reach a minimum period at $\sim 70 \mathrm{~min}$ and then begin to evolve back to longer orbital periods. As a CV approaches the minimum period, the mass of the donor star

* Based in part on observations made with the NASA/ESA Hubble Space Telescope, obtained at the Space Telescope Science Institute, which is operated by the Association of Universities for Research in Astronomy, Inc., under NASA contract NAS 5-26555, on observations made at the $1.2 \mathrm{~m}$ telescope, located at Kryoneri Korinthias, and owned by the National Observatory of Athens, Greece, and with the Isaac Newton Telescope and William Herschel Telescope, which are operated on the island of La Palma by the Isaac Newton Group in the Spanish Observatorio del Roque de los Muchachos of the Instituto de Astrofisica de Canarias.

$\star \star$ Visiting Astronomer, German-Spanish Astronomical Center, Calar Alto, operated by the Max-Planck-Institut für Astronomie, Heidelberg, jointly with the Spanish National Commission for Astronomy. decreases until it can no longer sustain hydrogen burning. The low mass secondary star becomes increasingly degenerate, entering the brown dwarf regime. Kolb (1993) estimated that 70\% of all CVs should have such "substellar" secondaries. Despite these large predicted numbers, only a handful of CVs are currently believed to harbour a brown dwarf donor, with WZ Sge, EG Cnc and EF Eri being among the best candidates (Littlefair et al. 2003; Patterson 1998; Beuermann et al. 2000). So far, for none of them does a compelling proof of the degenerate nature of the star exist. The discrepancy between the observed and predicted numbers of brown dwarf CVs could be due to selection effects: because of the low mass transfer rates predicted for these systems they may be substantially fainter than most CVs and may have very rare outbursts. Alternatively, it may also be that the standard theory is wrong, and that CVs have either not reached the period minimum where their donors become degenerate (King \& Schenker 2002), or they may even die before reaching the period minimum (Patterson 1998).

In order to be able to test properly any of these hypotheses we need to reduce the observational biases that afflict the 
Table 1. Log of observations.

\begin{tabular}{lcccc}
\hline \hline \multicolumn{5}{c}{ Photometry } \\
Date & UT time & Filter & Exp. [s] & \# Frames \\
\hline 40 cm Braeside Observatory & & & \\
2000 Sep. 25 & $04: 35-12: 29$ & $R$ & 50 & 510 \\
2000 Sep. 28 & $05: 26-10: 54$ & $R$ & 85 & 211 \\
2000 Oct. 03 & $02: 21-12: 01$ & $R$ & 95 & 338 \\
2001 Nov. 21 & $04: 13-08: 44$ & $\mathrm{C}$ & 85 & 152 \\
2001 Nov. 22 & $02: 43-08: 45$ & $\mathrm{C}$ & 55 & 302 \\
2003 Oct. 12 & $02: 19-11: 44$ & $\mathrm{C}$ & 55 & 552 \\
2003 Oct. 13 & $02: 36-11: 30$ & $\mathrm{C}$ & 55 & 542 \\
2003 Oct. 15 & $03: 56-11: 23$ & $\mathrm{C}$ & 55 & 446 \\
1.2 m Kryoneri Observatory & & & \\
2002 Oct. 15 & $18: 41-22: 39$ & $\mathrm{C}$ & 30 & 340 \\
2002 Oct. 16 & $17: 29-22: 13$ & $\mathrm{C}$ & 25 & 429 \\
2003 Aug. 14 & $22: 56-02: 52$ & $\mathrm{C}$ & 10 & 1029 \\
2003 Aug. 15 & $23: 14-02: 57$ & $\mathrm{C}$ & 10 & 1012 \\
2003 Aug. 16 & $23: 33-03: 06$ & $\mathrm{C}$ & 10 & 980 \\
2003 Aug. 17 & $23: 32-03: 04$ & $\mathrm{C}$ & 10 & 948 \\
2003 Aug. 18 & $22: 23-03: 01$ & $\mathrm{C}$ & 10 & 1249 \\
2003 Aug. 19 & $22: 09-03: 04$ & $\mathrm{C}$ & 10 & 1311 \\
2003 Aug. 20 & $21: 56-03: 07$ & $\mathrm{C}$ & 10 & 1432 \\
4 m WHT/ULTRACAM & & & \\
2003 Nov. 10 & $19: 23-22: 00$ & $u^{\prime}$ & 1 & 10013 \\
2003 Nov. 10 & $19: 23-22: 00$ & $g^{\prime}$ & 1 & 10013 \\
2003 Nov. 10 & $19: 23-22: 00$ & $r^{\prime}$ & 1 & 10013 \\
\hline
\end{tabular}

current $\mathrm{CV}$ population to a minimum. This is the main purpose of an undergoing large-scale search for CVs using the Hamburg Quasar Survey (HQS; Hagen et al. 1995; Gänsicke et al. 2000, 2002, 2004; Araujo-Betancor et al. 2003; Rodríguez-Gil et al. 2004). One of our most recent discoveries, HS $2331+3905$ (HS 2331 thereafter), was selected as a CV candidate on the basis of its spectral characteristics in the HQS. Specifically, its HQS spectrum displays the strong Balmer emission lines that suggest ongoing accretion in CVs.

In this paper we present the results of follow-up spectroscopy and photometry of HS 2331 obtained over three years, which confirm the CV nature of this object and unveil a very unusual picture. In Sect. 2 we describe the photometric and spectroscopic (ground and space) observations and data reduction and calibration. The analysis of the photometry and ground-based spectroscopy is presented in Sects. 3 and 4. In Sect. 4 we also present a model for the spectral energy distribution of HS 2331, from which we derive a temperature for the white dwarf and distance to the system. Finally, in Sect. 5, we use our findings in trying to construct a preliminary picture of the nature of HS 2331.

\section{Observations and data reduction}

\subsection{Photometry}

\subsubsection{Braeside observatory}

Differential $R$-band CCD photometry of HS 2331 was obtained at the Braeside Observatory immediately following the
Table 1. continued.

\begin{tabular}{lcccc}
\hline \hline \multicolumn{5}{c}{ Spectroscopy } \\
Date & UT time & Grating & Exp. [s] & \# Spectra \\
\hline 2.2 m Calar Alto/CAFOS \\
2000 Sep. 20 & $00: 50$ & B-200 & 600 & 1 \\
2000 Sep. 20 & $01: 05$ & R-200 & 600 & 1 \\
2000 Sep. 23 & $21: 20-02: 30$ & B-100 & 600 & 24 \\
2000 Sep. 24 & $00: 22-03: 37$ & B-100 & 600 & 16 \\
2000 Sep. 24 & $03: 47-04: 27$ & R-100 & 600 & 3 \\
2003 Sep. 11 & $22: 16-04: 20$ & G-100 & 600 & 28 \\
2003 Sep. 15 & $02: 28-04: 35$ & G-100 & 600 & 12 \\
2003 Sep. 16 & $23: 48-04: 04$ & G-100 & 600 & 23 \\
2.5 m Isaac Newton Telescope/IDS & & \\
2002 Aug. 26 & $02: 37-03: 60$ & R632V & 600 & 9 \\
2002 Aug. 28 & $00: 33-01: 56$ & R632V & 600 & 9 \\
2002 Sep. 01 & $04: 16-04: 47$ & R632V & 600 & 4 \\
2002 Sep. 02 & $03: 09-03: 41$ & R632V & 600 & 4 \\
Hubble Space Telescope/STIS & & \\
2002 Oct. 24 & $11: 34$ & G140L & 700 & 1 \\
2.4 m MDM Hiltner & & & \\
2003 Oct. 12 & $07: 28-08: 15$ & 600 & 420 & 7 \\
2003 Oct. 13 & $07: 38-08: 17$ & 600 & 420 & 6 \\
2003 Oct. 14 & $08: 03-08: 59$ & 600 & 420 & 8 \\
2003 Oct. 15 & $02: 51-10: 48$ & 600 & 420 & 21 \\
2003 Oct. 16 & $03: 45-04: 40$ & 600 & 420 & 8 \\
\hline
\end{tabular}

identification of its CV nature in September-October 2000 (Sect. 2.2.1), using a $0.4 \mathrm{~m}$ reflector equipped with a SITe 512 CCD camera (Table 1). HS 2331 was again observed from Braeside in November 2001 and October 2003, on both occasions in white light. The data were bias-subtracted, dark current-subtracted and flat-fielded in a standard fashion, and instrumental aperture magnitudes of HS 2331 were derived relative to USNO-A2.0 1275-18486676 ( $R=14.6$, labelled "C1" in Fig. 1). The mean magnitude of HS 2331 during the $2000 \mathrm{ob}-$ servations was $R \simeq 16.5$.

The Braeside CCD light curves of HS 2331 display periodic variability with an amplitude of $\sim \pm 0.1 \mathrm{mag}$ (Fig. 2 ), which has consistently been detected in all three years covered by our observations. The morphology of the photometric modulation is best described by a double-humped pattern with a period of $\sim 80 \mathrm{~min}$. The relative strength of the two humps varies substantially between the individual nights. The 2003 data reveal narrow absorption dips centred on some of the observed minima between the humps.

\subsubsection{Kryoneri observatory}

Filterless CCD photometry of HS 2331 was obtained during two nights in October 2002 and during 7 nights in August 2003 at the $1.2 \mathrm{~m}$ Kryoneri telescope using a SI-502 $516 \times$ 516 CCD camera (Table 1). The Kryoneri data were reduced following the procedure described in Gänsicke et al. (2004), and differential magnitudes were derived relative to $\mathrm{C} 1$ (Fig. 1).

The October 2002 light curves, obtained with a time resolution of $25 \mathrm{~s}-30 \mathrm{~s}$, were essentially similar to the Braeside 


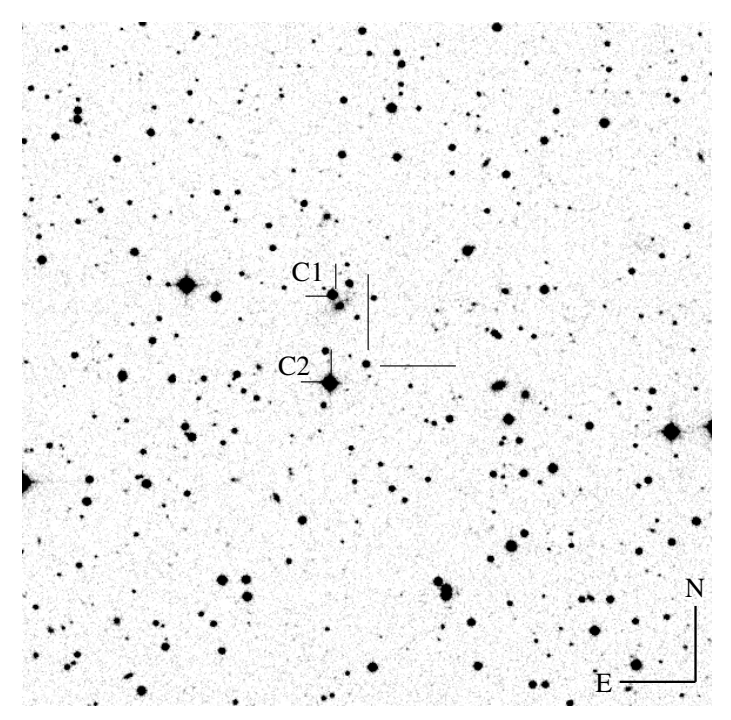

Fig. 1. $10^{\prime} \times 10^{\prime}$ Finding chart of HS 2331, obtained from the Digitized Sky Survey 2. The coordinates of the CV are $\alpha(2000)=23^{\mathrm{h}} 34^{\mathrm{m}} 01.6^{\mathrm{s}}$, $\delta(2000)=+39^{\circ} 21^{\prime} 41.4^{\prime \prime}$. Differential magnitudes of HS 2331 were obtained relative to the comparison stars C1 (USNO-A2.0 127518486676) and C2 (GSC 0323100595).

observations (Fig. 2), displaying a double-humped structure with a period of $\sim 80 \mathrm{~min}$. The higher time resolution data obtained in August 2003 provide stronger evidence for the absorption dips detected in the Braeside observations, and clearly reveal additional short-term variability on a time scale of $\sim 5$ min (e.g. August 14; Fig. 2). In addition the changes of the shape of the double-humped structure are more pronounced than in the Braeside observations. On August 19, one of the humps is extremely weak, on August 20 both humps have nearly equal amplitude.

\subsubsection{ULTRACAM on the William Herschel telescope}

The highest time resolution and signal-to-noise ratio CCD photometry was obtained on November 10, 2003 using the 3-beam ULTRACAM CCD camera (which takes CCD data in three colour channels simultaneously, Dhillon et al. 2002) on the William Herschel Telescope. HS 2331 was observed with a $1 \mathrm{~s}$ time resolution, using Sloan $u^{\prime}, g^{\prime}$, and $r^{\prime}$ filters. The data were reduced with the ULTRACAM pipeline. Differential magnitudes of HS 2331 were obtained relative to GSC 0323100595 (labelled "C2" in Fig. 1), and converted to apparent magnitudes using observations of the Sloan standard SA100-280, which was observed at the end of the night. The mean magnitudes of HS 2331 were $u^{\prime}=16.1, g^{\prime}=16.3$ and $r^{\prime}=16.0$. These values are subject to some small systematic uncertainty related to the yet uncalibrated colour terms accounting for the differences between the ULTRACAM and Sloan setups.

The ULTRACAM light curves impressively confirm the presence of variability on a time scale of $\sim 5 \mathrm{~min}$ seen in the Kryoneri data, as well as additional variability on even shorter time scales (Fig. 3).

\subsection{Optical spectroscopy}

\subsubsection{Calar Alto}

HS 2331 was identified as a CV from low-resolution spectroscopy obtained at the Calar Alto $2.2 \mathrm{~m}$ telescope on September 20, 2000. The CAFOS focal reductor spectrograph was used in conjunction with the standard SITe CCD. A single pair of blue/red identification spectra was obtained with the B-200 and R-200 grating through a 2" slit (see Table 1). The wavelength range of the combined spectra extends from 3500 to $10000 \AA$ at a spectral resolution of approximately $10 \AA$. The online reduction of the identification spectra using the CAFOS quick look context within MIDAS immediately revealed the CV nature of HS 2331 by the presence of broad double peaked Balmer and $\mathrm{He} \mathrm{I}$ emission lines in the spectra.

Time-resolved follow-up spectroscopy of HS 2331 was carried out during the same observing run, on September 23 and 24, 2000. A total of 43 spectra of 600 s each were obtained during the two nights, which were mainly photometric except for very thin cirrus during the last $\sim 1.5 \mathrm{~h}$ of the second night (Table 1). The B-100 grism was used along with a $1.5^{\prime \prime}$ slit to obtain 40 spectra with a useful wavelength range of 3500-6300 $\AA$ and a spectral resolution of $\sim 4 \AA$. In addition, 3 red spectra were acquired during the second night, using the R-100 grism with a $1.5^{\prime \prime}$ slit width. The red spectra cover a wavelength range of 6000 to $9200 \AA$ at a spectral resolution of $\sim 4 \AA$. Mercury-cadmium, helium-argon and rubidium arcs were taken regularly throughout both nights to provide for the wavelength calibration. Data from the flux standards G191B2B and $\zeta$ Cas were also obtained to correct for the instrument response of the "blue" and "red" spectra respectively. The CAFOS acquisition image were taken with a Johnson $V$ filter in order to correct the spectra for slit losses.

All spectra were reduced in a standard manner using the Figaro package within the Starlink software collection. The bias level was subtracted from the calibration and object images using the median from a set of unexposed frames. Dome flat field images, taken with the same set up as the object frames, were used to remove pixel to pixel variations. The spectra were then optimally extracted (Horne 1986) and sky line subtracted. A fourth-order polynomial was fitted to obtain the dispersion relation for each of the arc spectra, giving an rms smaller than $0.1 \AA$ in all cases. Each target spectrum was then wavelength-calibrated by interpolating two neighboring arc spectra to account for the possible drift of the wavelengthto-pixel scale. The instrumental response function was computed as the ratio of a spline fit to the line-free continuum of the B-100/R-100 spectra of the flux standards to the fluxcalibrated data from Filippenko \& Greenstein (1984) and Oke (1990). All target spectra were flux-calibrated and corrected for atmospheric extinction using this response function.

We used the acquisition images obtained in conjunction with the CAFOS spectroscopy to estimate the brightness of HS 2331 during our Calar Alto observations. Aperture photometry relative to GSC $0323100595(V=12.75)$ located $\sim 35$ " south-east of HS 2331 ("C2" in Fig. 1) resulted in $V \simeq 16.4$, which is consistent with the magnitude obtained 

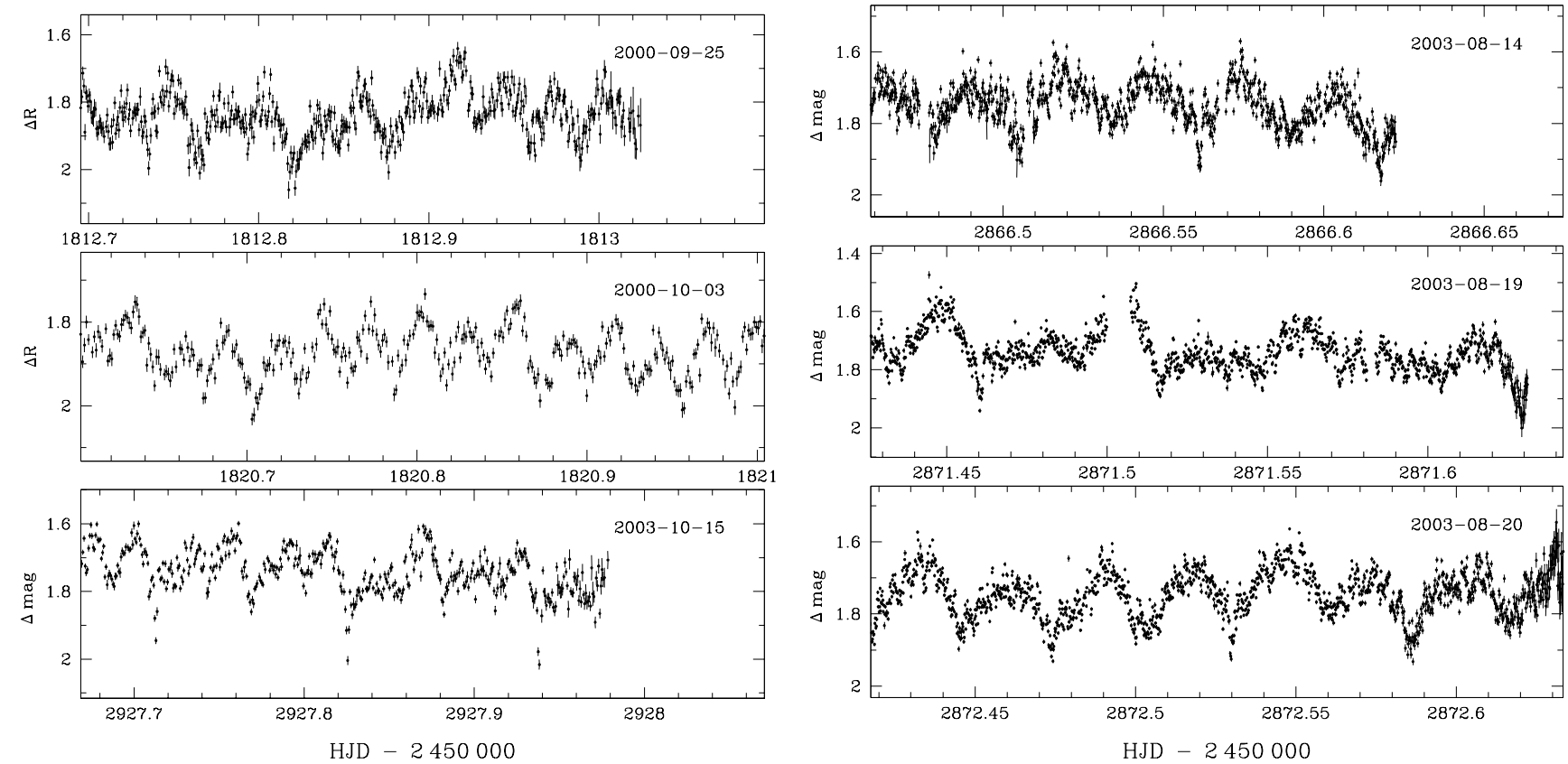

Fig. 2. Sample light curves of HS 2331 based on differential CCD photometry obtained at the $40 \mathrm{~cm}$ Braeside Observatory (left) and at the $1.2 \mathrm{~m}$ Kryoneri Observatory (right).

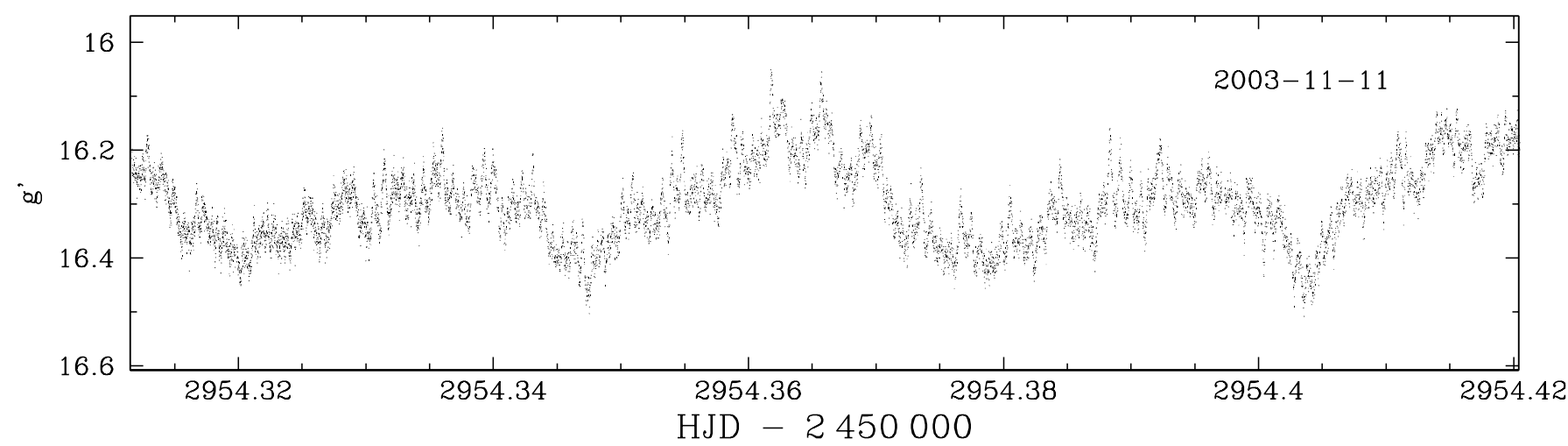

Fig. 3. Light curve of HS 2331 obtained at a time resolution of $1 \mathrm{~s}$ with the $g^{\prime}$-filter using ULTRACAM on the WHT.

from the HQS direct plate, and with the brightness level of all our photometry (Sect. 2.1). A grand average spectrum of HS 2331 was created by combining the mean of all "blue" and "red" spectra from the flux-calibrated (and corrected for atmospheric extinction) B-100/R-100 data set. This average spectrum was then scaled to $V=16.4$, as determined from the CAFOS acquisition images to account for possible slit losses (Fig. 4). The applied correction of $\simeq 0.2 \mathrm{mag}$ is comparable to the intrinsic variability of HS 2331, and indicates that the absolute error in our flux calibration is $\leq 20 \%$.

A further set of observations were obtained in September 2003 at the $2.2 \mathrm{~m}$ Calar Alto telescope. This time the G-100 grism was used which together with a slit width of 1.2" provided a wavelength range of 4240-8300 $\AA$ and a spectral resolution of $\sim 4.1 \AA$ (Table 1 ). These spectra were reduced in an analogous fashion as described above.

The spectrum of HS 2331 (Fig. 4) displays broad doublepeaked Balmer and $\mathrm{He}$ I emission lines, suggesting an origin in the accretion disc. The higher series of the Balmer lines are

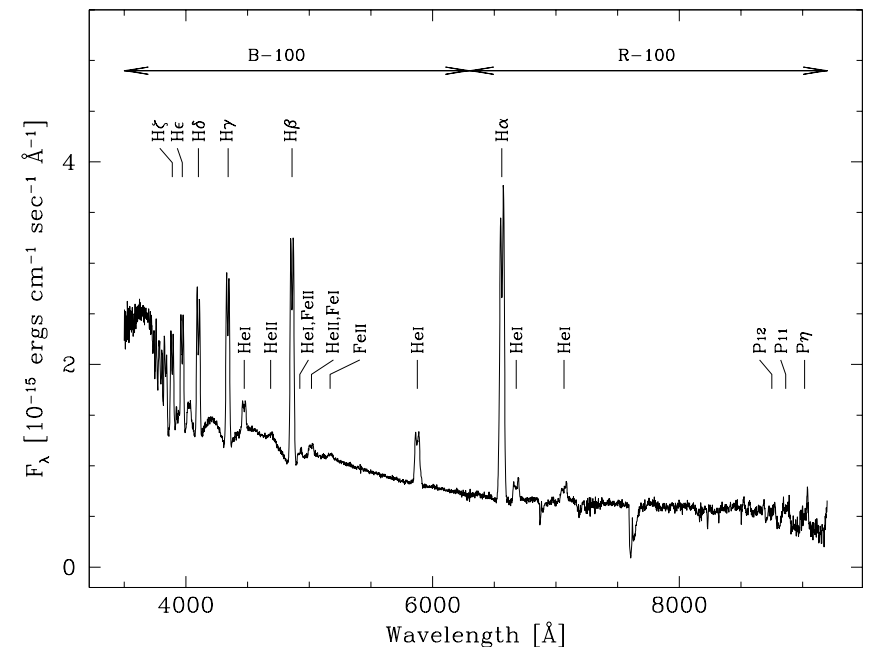

Fig. 4. Mean, flux-calibrated "blue" and "red" spectra (Calar Alto 2000, B-100/R-100 data set) of HS 2331 combined together. The arrows indicate the wavelength range covered by the B-100 and R-100 gratings. 
Table 2. $E W$ and $F W H M$ (corrected for the instrumental resolution) of the strongest Balmer emission lines and of the strongest FUV lines.

\begin{tabular}{lrcc}
\hline \hline Line & $E W[\AA]$ & $F W H M\left[\mathrm{~km} \mathrm{~s}^{-1}\right]$ & $F W H M[\AA]$ \\
\hline $\mathrm{H} \alpha$ & $185 \pm 14$ & $1312 \pm 5$ & $28.7 \pm 0.1$ \\
$\mathrm{H} \beta$ & $75 \pm 9$ & $1475 \pm 6$ & $23.9 \pm 0.1$ \\
$\mathrm{H} \gamma$ & $40 \pm 4$ & $1583 \pm 7$ & $22.9 \pm 0.1$ \\
$\mathrm{H} \delta$ & $30 \pm 13$ & $1403 \pm 7$ & $19.2 \pm 1.1$ \\
$\mathrm{C}$ IV & $234 \pm 14$ & $2478 \pm 19$ & $12.8 \pm 0.1$ \\
$\mathrm{Si}$ IV & $52 \pm 13$ & $3749 \pm 236$ & $17.5 \pm 1.1$ \\
$\mathrm{C}_{\text {II }}$ & $67 \pm 12$ & $2476 \pm 68$ & $11.0 \pm 0.3$ \\
$\mathrm{C}$ III & $111 \pm 19$ & $2500 \pm 51$ & $9.8 \pm 0.2$ \\
$\mathrm{~N} \mathrm{~V}$ & $42 \pm 15$ & $2417 \pm 48$ & $10.0 \pm 0.2$ \\
\hline
\end{tabular}

flanked by extremely broad absorption troughs, which are reminiscent of the Stark-broadened absorption lines observed in the high-gravity atmospheres of cool white dwarfs (the width of the absorption lines are too wide to be of accretion disc origin). The red part of the spectrum does not contain any spectral features that could be ascribed to the emission of the secondary star.

Table 2 lists the equivalent widths $(E W)$ and full width at half maximum ( $F W H M$, corrected for the instrumental response), of $\mathrm{H} \alpha-\mathrm{H} \delta$. The $E W$ and the FWHM measurements were obtained by fitting a single Gaussian in all cases. Average spectra were used for the Balmer lines: Calar Alto 2000 for $\mathrm{H} \delta$ and Calar 2003 for $\mathrm{H} \alpha-\mathrm{H} \delta$.

\subsection{2. $2.5 \mathrm{~m}$ Isaac Newton telescope}

A total of 26 spectra of $600 \mathrm{~s}$ each, spread out over a week, were obtained in August-September 2002 (Table 1). We used the Intermediate Dispersion Spectrograph (IDS) on the $2.5 \mathrm{~m}$ Isaac Newton Telescope (INT), at the Roche de los Muchachos observatory in La Palma. The IDS was equipped with the R632V grating and the $2048 \times 4100$ pixel EEV10 detector. Using a slit width of $1.5^{\prime \prime}$ the setup provided an unvignetted wavelength range of $\sim 4400 \AA-6800 \AA$ and a spectral resolution of $\sim 2.3 \AA$. Copper-Argon wavelength calibrations (arcs) were obtained at the beginning and end of each observation block of HS 2331. The reduction procedure applied to this data set is identical to the one applied to the Calar Alto/CAFOS data above.

\subsection{3. $2.4 \mathrm{~m}$ Hiltner telescope}

Most recently, we observed HS 2331 from the $2.4 \mathrm{~m}$ Hiltner telescope at MDM observatory (Table 1). We obtained a total of 50 spectra of $420 \mathrm{~s}$ each in October 2003. The modular spectrograph combined with the 600 line $\mathrm{mm}^{-1}$ grating and a SITe $2048 \times 2048$ CCD detector, yielded a spectral dispersion of $\sim 2.0 \AA \mathrm{pixel}^{-1}$ from 4000 to $7500 \AA$. The reduction technique applied to this set of data is as explained in Thorstensen et al. (1998).

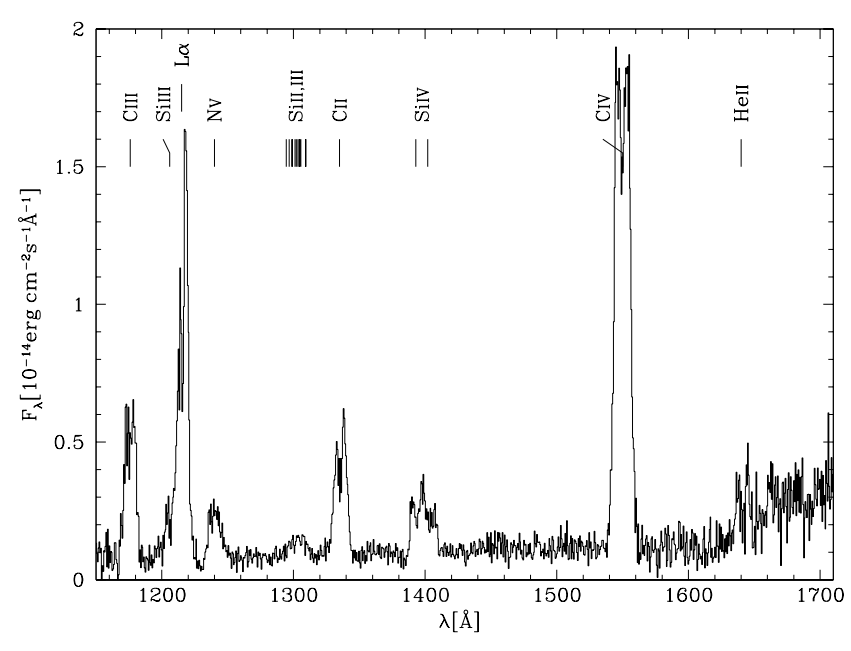

Fig. 5. HST/STIS spectrum of HS 2331.

\subsubsection{Hubble space telescope}

Far-ultraviolet $(F U V)$ spectroscopy of HS 2331 was obtained with the Hubble Space Telescope/Space Telescope Imaging Spectrograph (HST/STIS) on October 24, 2002 as part of an ongoing snapshot survey of CVs. The observations were obtained using the G140L grating and the $52^{\prime \prime} \times 0.2^{\prime \prime}$ aperture, providing a spectral resolution of $R \approx 1000$ over the wavelength range $1150-1710 \AA$. The data were pipeline-processed within IRAF using CALSTIS V2.13b. The F28 $\times$ 50LP magnitude of HS 2331 measured from the STIS CCD acquistion image confirms that the system was at its usual brightness level during the FUV observations. The STIS spectrum (Fig. 5) contains the mixture of low and high-ionisation emission lines often observed in CVs: C III $\lambda$ 1176, Si III $\lambda 1206$, Nv $\lambda \lambda 1239,43, C_{\text {II }} \lambda 1335$, a broad complex of Si III near $1300 \AA, \quad S i \operatorname{IV} \lambda \lambda 1394,1403, C_{\text {IV }} \lambda \lambda 1548,51$ and probably $\mathrm{He}$ II $\lambda$ 1640. The emission line flux ratios are within the normal range observed in CVs (Mauche et al. 1997; Gänsicke et al. 2003). Several of the lines contained in the STIS are doublepeaked, again suggesting an origin in an accretion disc. The $E W$ and $F W H M$ of the strongest lines in the STIS spectrum were estimated by fitting a Gaussian to the observed line profiles, and the results are reported in Table 2. Correcting the FWHM for the separation of the $\mathrm{C}_{\mathrm{II}}, \mathrm{C}$ IV and $\mathrm{Si}$ IV doublets results in line widths which are comparable to those of the Balmer lines, suggesting a common origin of the FUV and optical emission lines.

The continuum flux underlying the emission lines is slightly increasing towards the red, with a sudden upturn in flux at wavelengths $\lambda \gtrsim 1650 \AA$.

\section{Analysis: Photometry}

We have analysed the complex variability detected in the light curves of HS 2331 (for a small sample see Figs. 2 and 3) by computing Scargle periodograms (Scargle 1982) for the entire CCD photometry of HS 2331, as well as for individual subsets of the data, using the MIDAS/TSA context. Prior to this period analysis, we have subtracted the nightly mean of each 

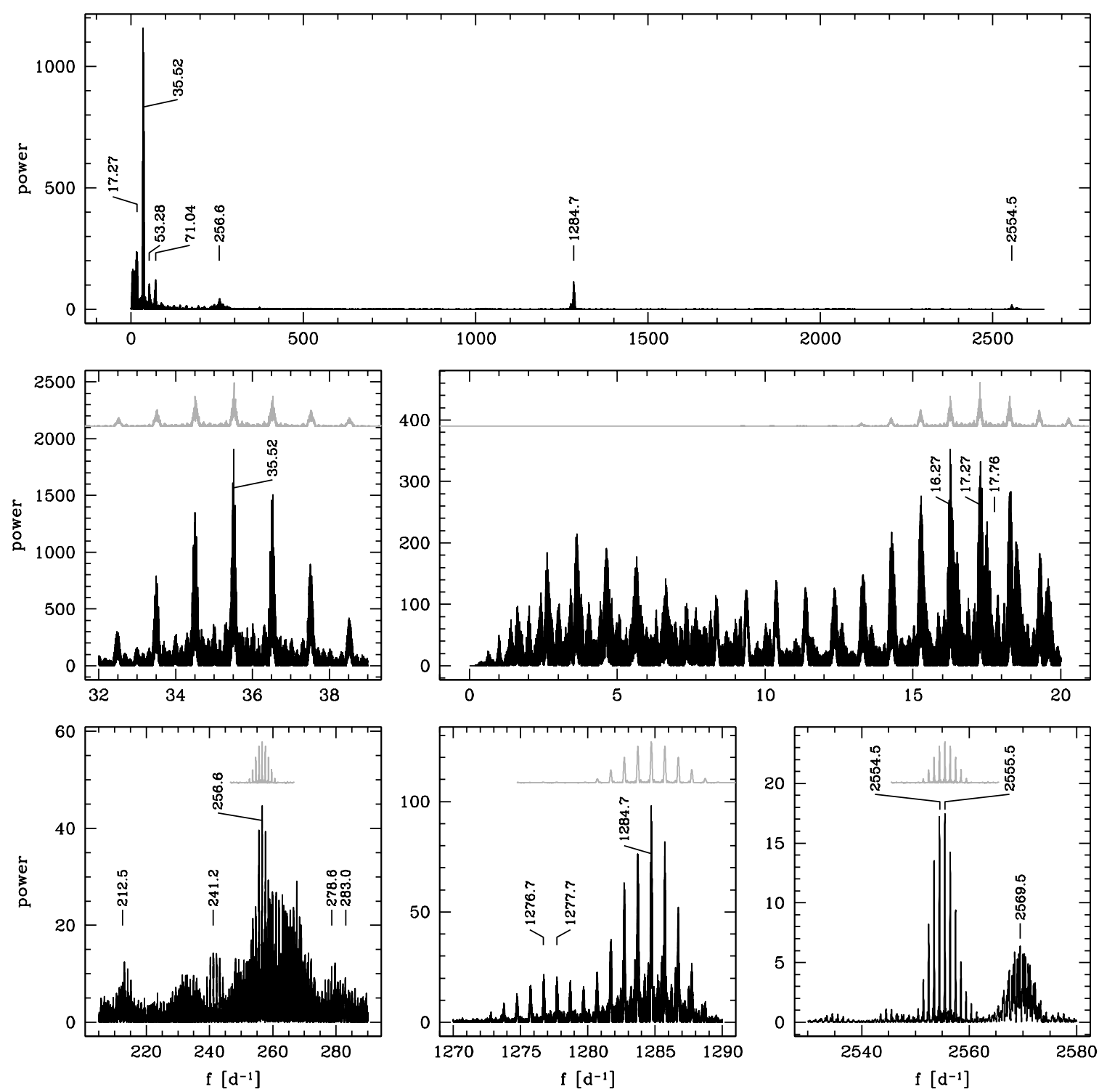

Fig. 6. Scargle periodograms computed from the CCD photometry of HS 2331. Top panel: the entire frequency range sampled by the Kryoneri 2003 data, which represents the largest and best sampled data set to investigate periodicities on time scales of $\sim 2 \mathrm{~d}$ to $\sim 30 \mathrm{~s}$. Middle panels: close-ups of the power spectra calculated from all available photometric data (Table 1). The left panel shows a coherent signal at $35.52 \mathrm{~d}^{-1}$. Shown in gray is the window function of the entire data set, shifted to the frequency of the strongest peak. We interpret this signal as twice the orbital frequency, as the orbital light curve is dominated by a double-hump structure (Fig. 7). The right panel shows several signals in the low-frequency range. Practically no power is detected at the orbital frequency (i.e. $17.76 \mathrm{~d}^{-1}$ or 81.08 min), but a strong signal is found at $17.27 \mathrm{~d}^{-1}(83.38 \mathrm{~min})$ and the one-day alias at $16.27 \mathrm{~d}^{-1}(88.51 \mathrm{~min})$. Bottom panels: close-up views of the high-frequency signals in the Scargle periodogram computed from the Kryoneri 2003 data only. As in the middle panels, the gray line shows the alias structure resulting from the sampling of our observations. Clear signals are detected at $5.61 \min \left(256.6 \mathrm{~d}^{-1}\right), 1.12 \min \left(1284.7 \mathrm{~d}^{-1}\right)$, and $0.56 \mathrm{~min}\left(2555.5 \mathrm{~d}^{-1}\right)$.

light curve to account for night-to-night variations in the overall brightness of the system and more importantly of the different detectors/filters used during the observations. The periodogram of HS 2331 calculated from the Kryonery 2003 data, the largest and best sampled photometric data set, (Fig. 6; top panel) contains a multitude of signals, as anticipated by our visual inspection of the light curves (Sect. 2.1).

The strongest peak is located at $35.52 \mathrm{~d}^{-1}$ (40.54 min), a weaker signal is centred on $\sim 17.27 \mathrm{~d}^{-1}$ (83.38 $\mathrm{min}$ ), and some power is also found at $53.28 \mathrm{~d}^{-1}(27.03 \mathrm{~min})$ and $71.04 \mathrm{~d}^{-1}$ (20.27 $\mathrm{min})$. In the high-frequency range there is a strong peak at $1284.7 \mathrm{~d}^{-1}(1.12 \mathrm{~min})$ and a weaker one at $256.6 \mathrm{~d}^{-1}$ (5.61 min).

\subsection{The orbital period}

Figure 6 shows a close-up view of the Scargle periodogram of the $\sim 40$ min signal (middle left panel). The strongest peak is located at $40.542632 \pm 0.000008 \min \left(35.518168 \pm 0.000007 \mathrm{~d}^{-1}\right)$ with the one $\sigma$ error obtained from a sine fit to the entire photometric data. The other peaks seen in the periodogram are aliases separated by multiples of $1 \mathrm{~d}^{-1}$. The sharpness and power of 


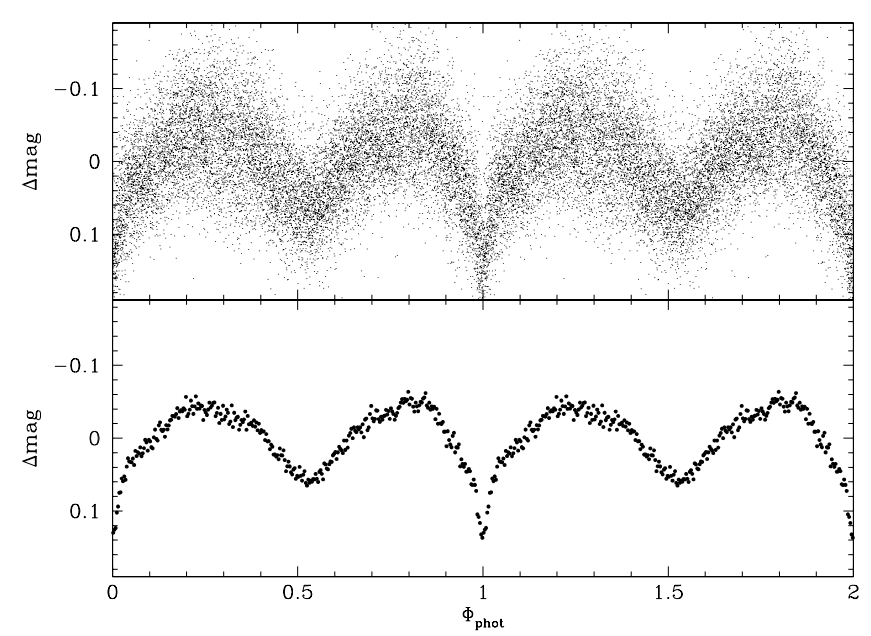

Fig. 7. HS 2331 entire photometric data set folded over $81.08 \mathrm{~min}$ (upper panel) and re-sampled into bins of 100 points (bottom panel). The light curves are repeated over two cycles for clarity.

the 40.54 min signal indicates a very high degree of coherence, suggesting that it is related to a stable "clock" within the binary system, such as the binary orbital period or the white dwarf spin period.

Folding the entire 4 years worth of photometry over exactly twice the $40.54 \mathrm{~min}$ signal, i.e. $81.08 \mathrm{~min}$, results in an average light curve (Fig. 7) that unambiguously recreates the morphology observed in the individual light curves (Figs. 2 and 3): a double-humped modulation with a narrow dip. Dips of such shape are observed either in strongly magnetic CVs, when the magnetically funnelled accretion stream passes in front of the white dwarf, or as grazing eclipses of the hot spot in the accretion disc in non-magnetic CVs. Stream eclipses in magnetic CVs are relatively unstable features as the location of the stream within the binary frame changes as a function of the mass transfer rate and therefore we favour the latter scenario as the cause of the narrow dips.

The light curve of HS 2331 (Figs. 2 and 7) bears a striking resemblance to that of one of the most (in)famous and thoroughly studied short period CVs: WZ Sge (see e.g. Fig. 1 of Patterson et al. 1998). Both objects exhibit double humps in their light curves, in both cases one of the humps appear to suffer from sporadic absorption events, and both systems show a sharp and relatively shallow eclipse. From the strict coherence of the $40.54 \mathrm{~min}$, the detection of periodically recurrent eclipses, and the close analogy to WZ Sge, we conclude that the orbital period of HS 2331 is $81.08 \mathrm{~min}$, and we define the following eclipse ephemeris

$\phi_{0}=\operatorname{HJD} 2451812.67765(35)+0.05630921(1) \times E$

where phase zero corresponds to the time of mid eclipse. Higher harmonics of the orbital period are clearly visible in the periodogram (Fig. 6) at $27.03 \mathrm{~min}\left(53.28 \mathrm{~d}^{-1}\right)$ and $20.27 \mathrm{~min}$ $\left(71.04 \mathrm{~d}^{-1}\right)$.

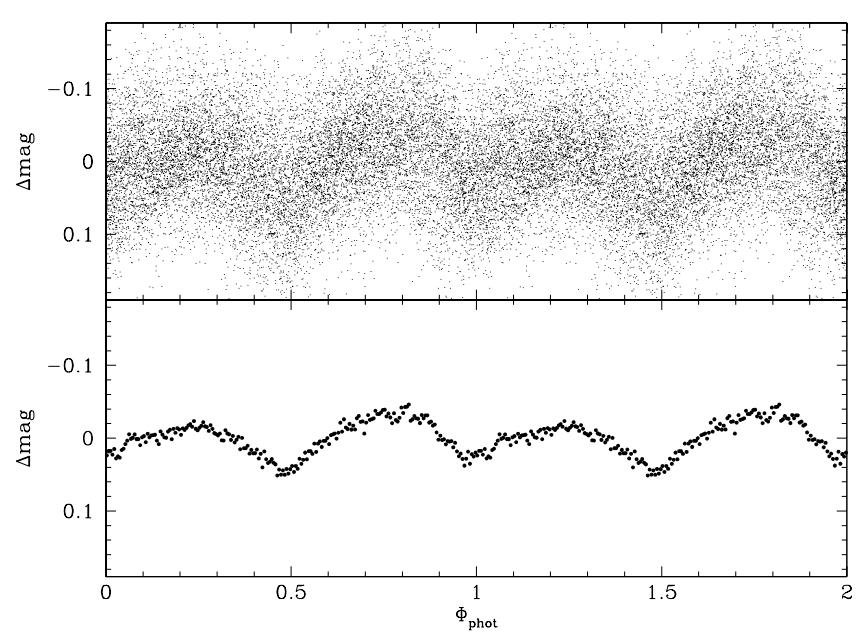

Fig. 8. HS 2331 entire photometric data set folded over $83.38 \mathrm{~min}$ (upper panel) and re-sampled into bins of 100 points (bottom panel). The light curves are repeated over two cycles for clarity.

\subsection{Quiescent superhumps?}

Only a weak signal is detected at the orbital period of $81.08 \mathrm{~min}$ (Fig. 6, middle right). This is not too much of a surprise because of the double-humped structure of the orbital modulation, which shifts most power to half the orbital period. Surprising is, however, the detection of a relatively strong signal close to $P_{\text {orb }}$, with a period of either $\simeq 83.38 \mathrm{~min}\left(\simeq 17.27 \mathrm{~d}^{-1}\right)$ or $\simeq 88.51 \mathrm{~min}$ $\left(\simeq 16.27 \mathrm{~d}^{-1}\right)$. Both these 1-day aliases are of similar strength, and their relative power varies between different observing runs. In contrast to the $35.52 \mathrm{~d}^{-1}$ orbital signal (Fig. 6, middle left), the periodogram around the $83.38 \mathrm{~min} / 88.51 \mathrm{~min}$ signals is extremely complex, displaying significant substructure in excess to the window function, suggesting either the presence of more than one periodicity and/or a lower degree of coherence. Figure 8 shows the light curve folded over the $83.38 \mathrm{~min}$ signal detected in the periodogram of Fig. 6 (middle-left panel). The absence of eclipses in this folded light curve clearly rules out the 83.38 min signal (and its aliases) being the orbital period.

The separation of the $83.38 \mathrm{~min}$ signal and the orbital period of $81.08 \mathrm{~min}$ is conspicuously close to those observed for the superhump period in a large number of short period dwarf nova (e.g. Patterson 2001). In fact, the period excess found in HS 2331, $\epsilon=\left(P_{\mathrm{sh}}-P_{\mathrm{orb}}\right) / P_{\mathrm{orb}}=0.028$, is similar to that observed in LL And, which has an orbital period close to that of HS 2331. Based on the observational analogies between HS 2331 and a number of well-observed short-period dwarf novae, it is tempting to ascribe the observed $83.38 \mathrm{~min}$ signal to a permanent superhump. If this hypothesis were true, HS 2331 would be unique, as it displays a permanent quiescent superhump. If $88.51 \mathrm{~min}$ were the true period its interpretation in terms of a permanent superhump would result in $\epsilon=0.092$, much larger than any period excess observed in short period CVs, and we might as well have discovered a hitherto unknown phenomenon. 
Table 3. High-frequencies signals detected in the photometry of HS $2331+3905$.

\begin{tabular}{crrrrr}
\hline \hline \multicolumn{2}{c}{ Kryoneri } & \multicolumn{4}{c}{ WHT } \\
$\begin{array}{c}f \\
{\left[\mathrm{~d}^{-1}\right]}\end{array}$ & $\begin{array}{r}\text { clear } \\
{[\mathrm{mmag}]}\end{array}$ & \multicolumn{1}{c}{$f$} & \multicolumn{1}{c}{$u^{\prime}$} & \multicolumn{1}{c}{$\left.\mathrm{d}^{-1}\right]$} & \multicolumn{1}{c}{$r^{\prime}$} \\
\hline 205.7 & 3.6 & 206 & 9.6 & 6.7 & 7.4 \\
212.5 & 4.5 & 216 & 15.0 & 10.1 & 11.2 \\
213.2 & 4.8 & & & & \\
& & 226 & 14.2 & 11.9 & 12.1 \\
241.2 & 6.4 & & & & \\
256.6 & 10.0 & 266 & 36.3 & 20.2 & 22.1 \\
278.6 & 4.9 & & & & \\
283.0 & 3.6 & 288 & 11.7 & 7.7 & 8.6 \\
& & 474 & 6.6 & 5.3 & 4.8 \\
& & 535 & 7.2 & 6.6 & 6.5 \\
1029.1 & 2.6 & 1021 & 8.5 & 6.8 & 6.9 \\
1276.7 & 7.0 & & & & \\
1284.7 & 15.8 & 1283 & 35.1 & 20.7 & 20.6 \\
$2554.5^{(\dagger)}$ & 6.2 & 2554 & 7.6 & 9.4 & 6.2 \\
2569.5 & 3.7 & 2570 & 11.0 & 4.4 & 6.2 \\
\hline
\end{tabular}

(†) The 1-day aliases at 2555.5 has nearly equal power.

\subsection{Higher frequencies: White dwarf pulsations and spin?}

As mentioned above, the periodogram of HS 2331 contains a number of distinct signals at high frequencies (Fig. 6), most pronounced at $5.61 \mathrm{~min}\left(256.6 \mathrm{~d}^{-1}\right)$ and $1.12 \min \left(1284.7 \mathrm{~d}^{-1}\right)$. We have inspected in more detail the periodogram computed from the 2003 Kryoneri data, which provides the longest coverage at a high time resolution, as well as from the ULTRACAM observations, which has the highest time resolution but covers only $\sim 2.5 \mathrm{~h}$. Table 3 lists the frequencies and amplitudes of all signals that are unambiguously detected in the two data sets. The fact that most signals are detected over long periods of time rules out the CV-typical flickering to explain their origin.

The extremely complex power spectrum in the range around $\sim 5 \mathrm{~min}$ is indicative of a combination of many frequencies. Such a structure is reminiscent of the patterns detected in the power spectra of ZZ Ceti stars, non-radially pulsating white dwarfs with a hydrogen-rich atmosphere. They are found in a narrow range of effective temperatures, $12500 \mathrm{~K} \geq$ $T_{\text {eff }} \geq 10700 \mathrm{~K}$, and show multiperiodic variations with amplitudes reaching up to $\sim 0.3 \mathrm{mag}$ and periods ranging from $\sim 100$ to $\sim 1200$ s (Clemens 1993). Resolving the rich frequency spectrum of ZZCeti stars related to the various pulsation modes, their harmonics, and various linear combinations of modes requires alias-free uninterrupted multi-longitude observing campaigns (e.g. Kepler et al. 2003). In the case of HS 2331, the sampling of our 2003 Kryoneri run introduces too much of an alias structure (Fig. 6) to allow a secure identification of all the various frequencies.
Whereas our short ULTRACAM run is not very useful in identifying the frequencies present in HS 2331, these data show that the power of the $\sim 5$ min variability increases towards the blue, which is what would be expected for ZZ Ceti pulsations. Untypical for ZZ Ceti pulsations are the additional large-amplitude signals detected at extremely high frequencies (Fig. 6). The shortest period observed in any ZZ Ceti star is $70.9 \mathrm{~s}$ in G 185-32 (Castanheira et al. 2004). Our detection of power at the first harmonic of the $1.12 \mathrm{~min}$ signal seems to rule out a conventional $\mathrm{ZZCet}$ pulsation as the origin of the high-frequency signals. Yet another stable clock available in a $\mathrm{CV}$ is the spin of the white dwarf, and we tentatively suggest that the $1.12 \mathrm{~min}$ and $0.56 \mathrm{~min}$ signals are related to the white dwarf spin period and its first harmonic, respectively. Intriguing is the doublet-like structure of the $1.12 \mathrm{~min}$ and $0.56 \mathrm{~min}$ signals (Fig. 6). Fast rotation of the white dwarf will result in extremely non-linear perturbations of the pulsation frequencies, and may be responsible for this structure. Alternatively, the doublet-structure may reflect a sideband frequency, as typically observed in intermediate polars. The choice of the lower frequency alias in the doublets is somewhat ambiguous: assuming that $1277.7 \mathrm{~d}^{-1}$ and $2555.5 \mathrm{~d}^{-1}$ are the true frequencies of the doublet and its first harmonic, the implied beat frequency is $7 \mathrm{~d}^{-1}$, which is nearly identical to the spectroscopic period found from the radial velocity variations of the Balmer emission line wings (Sect. 4).

\section{Analysis: Spectroscopy}

\subsection{Radial velocities}

Radial velocities were measured using the convolution technique first outlined by Schneider \& Young (1980) and developed by Shafter (1983).

The emission line profile is convolved with two Gaussians of equal FWHM (of the order of the wavelength resolution) but with opposite signs. The peaks of the Gaussians are separated by a distance chosen in order to measure as far out as possible into the wings, while still retaining an adequate signal-to-noise level. By doing this we ensure that the measured radial velocities trace the inner part of the accretion disc, where one may hope to find an azimuthally symmetric structure that traces the orbital motion of the white dwarf. Prior to the actual radial velocity measurement the spectra were transformed into the heliocentric rest frame.

\subsubsection{The end of a myth...}

Radial velocity variations were measured for each individual observing run (Table 1) using the strongest Balmer lines as well as $\mathrm{He}$ I $\lambda 5875$ and $\mathrm{He}$ I 4471. In all cases the Gaussians separation was set to values between $2600 \mathrm{~km} \mathrm{~s}^{-1}$ and $3000 \mathrm{~km} \mathrm{~s}^{-1}$. The radial velocity measurements were then subjected to the Scargle period analysis in the MIDAS/TSA context. The results for each individual run did not depend on the emission line used for the analysis, and we will discuss in the following the radial velocity variations measured in $\mathrm{H} \gamma$ (Calar Alto 2000; we 

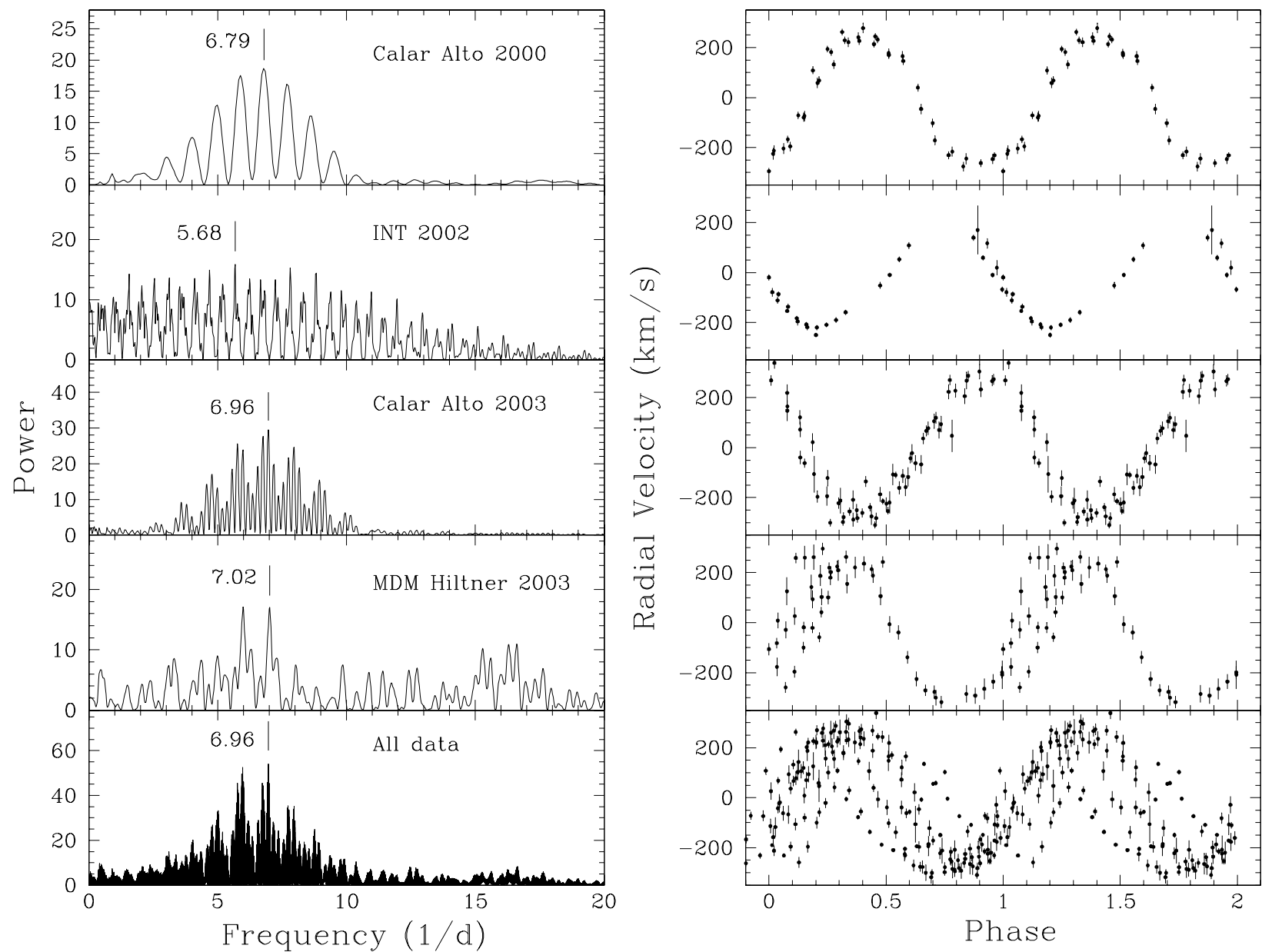

Fig. 9. Left panel: scargle periodograms of the individual data sets (see Table 1) and of all the data combined together. Right panel: radial velocity curves of the individual data sets and of all the data sets combined together, folded over the highest peak in their respective Scargle periodograms (shown on the left). The zero phase is taken to be the first point in each of the data sets respectively and the curves are repeated twice for clarity.

opted against $\mathrm{H} \beta$ because of the potential contamination from the nearby $\mathrm{He}$ lines) and $\mathrm{H} \alpha$ for all the other runs.

Figure 9 shows the periodograms and the corresponding radial velocity curves folded over the highest peaks detected in each of the data sets. The bottom panel shows the periodogram and folded radial velocity curve obtained when combining all data. The Calar Alto 2000 data show a smooth sinusoidal radial velocity variation with an amplitude of $\sim 220 \mathrm{~km} \mathrm{~s}^{-1}$ and a period of $3.53 \mathrm{~h}$, which is not anywhere close to the orbital period determined from the eclipses! This makes HS 2331, to our knowledge, the first $\mathrm{CV}$ in which the dominant radial velocity variation is not modulated on the orbital period. With HS 2331, there are now four CVs in which the photometric modulation and the spectroscopic modulation are significantly different; the other three being FS Aur, GW Lib and Aqr1 (see Table 2 of Woudt et al. 2004). The origin of the second period in these systems is much of a mystery as the $\sim 3.5 \mathrm{~h}$ periodicity in HS 2331, but contrary to HS 2331, in FS Aur, GW Lib and Aqr1 the spectroscopic variation is believed to be orbital. Figure 10 shows the trailed spectra of $\mathrm{H} \alpha$ and $\mathrm{H} \beta$ obtained from the observing night with the largest data set (i.e. Calar Alto 2003, Sep. 11; Table 1) in minutes and phase folded with the orbital period respectively. The $3.45 \mathrm{~h}$ period is clearly seen in the wings of the Balmer emission lines.

The analysis of the other runs reveals that the radial velocity variation changes on time scales of several days. The structure of the periodograms becomes increasingly complicated with the length of the run, and folding the radial velocity measurements over the strongest peak (or any other peak) will not provide a smooth radial velocity variation (Fig. 9). The strongest signals in the periodograms are found at $4.22 \mathrm{~h}$ (INT 2002), $3.45 \mathrm{~h}$ (Calar Alto 2003), 3.42 h (MDM Hiltner 2003) and $3.45 \mathrm{~h}$ (all data). All runs, with the exception of the INT data, give very close period values which appear consistent with each other. Close inspection of the bottom panel of Fig. 9 shows that, when folded over the $\sim 3.45 \mathrm{~h}$ signal, (the highest peak detected in the periodogram calculated from combining all the spectroscopic data together) a shift between the individual nights appears (Calar Alto 2000/2003 and MDM 2003 data). In the case of the INT observing run, the poor sampling of the data (Table 1) may prevent us from detecting the true underlying periodicity at this epoch and the relatively smooth appearance of the radial velocity curve folded over $4.22 \mathrm{~h}$ (the highest peak in the periodogram) may be coincidental. Statistically, there is 

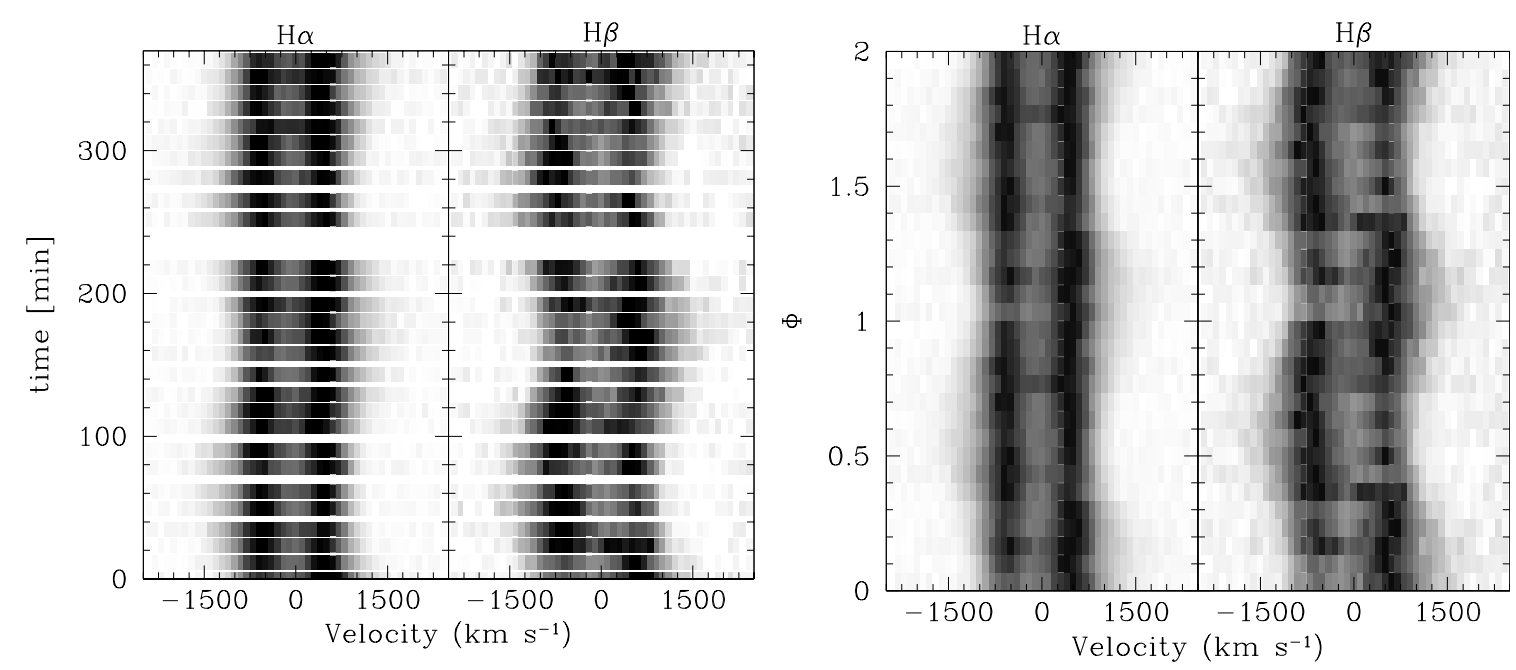

Fig. 10. From left to right: trail spectra of $\mathrm{H} \alpha$ and $\mathrm{H} \beta$ computed from the largest data set within an observing night (i.e. Calar Alto 2003, Sep. 24; see Table 1), and the same $\mathrm{H} \alpha$ and $\mathrm{H} \beta$ trail spectra but this time folded over the orbital period (i.e. $P_{\text {orb }}=81.08$ min). The white stripes in the trail spectra of the left panel are caused by lack of observations during the corresponding times.
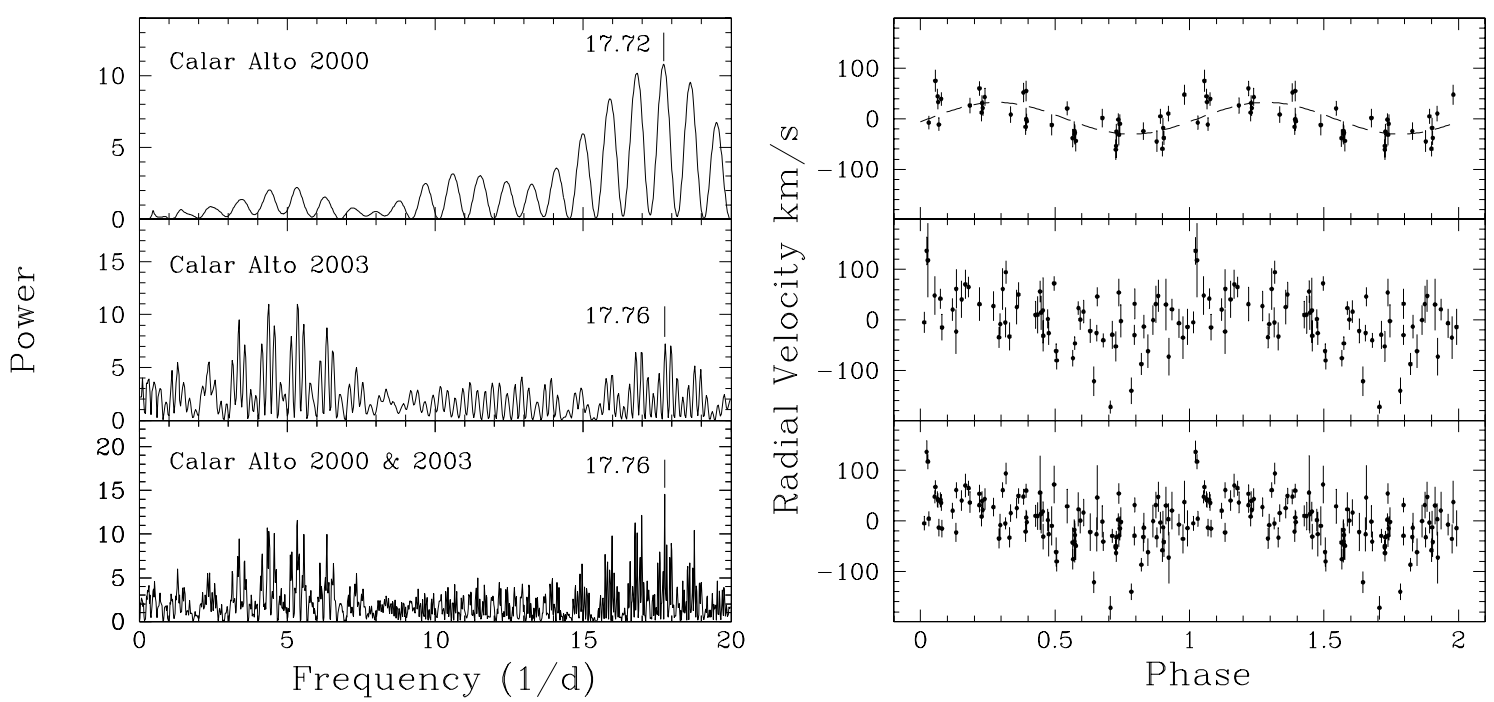

Fig. 11. Left panel: from top to bottom, Scargle periodograms of the detrended Calar Alto 2000 and Calar Alto 2003 spectroscopic data sets and a combination of both sets (i.e. Calar Alto 2000, 2003). The radial velocity measurements were detrended by subtracting a sine wave with their respective highest peak value (see the left panel of Fig. 9) as the frequency. The combined Calar Alto (2000, 2003) Scargle periodogram in the bottom panel was computed after detrending the data sets individually. Right panel: detrended radial velocity curves folded over the orbital period obtained from the photometry in Sect. 3 (i.e. $17.76 \mathrm{~d}^{-1}$ or $81.08 \mathrm{~min}$ ). The zero phase in these curves corresponds to the photometric zero phase as defined in Sect. 3 and the curves are repeated twice for clarity. Also shown on the top right panel is the best fit to the detrended radial velocity curve (see text for more details).

no strong preference for any of the aliases found in the range $\sim 4 \mathrm{~d}^{-1}-10 \mathrm{~d}^{-1}$.

We conclude that a persistent large-amplitude radial velocity variation with a period of $\sim 3.5 \mathrm{~h}$ is present in HS 2331 , however, this variation is not coherent and its period and/or phase drifts on time scales of days.

\subsection{2. ...sanity refound}

The fact that the dominant radial velocity variation in HS 2331 does not occur on the orbital period is most surprising, and alarming, as radial velocity variations measured from the emission lines are routinely used not only to determine the orbital periods of $\mathrm{CVs}$, but also to estimate the radial velocity of the white dwarf. In order to probe for lower amplitude radial velocity variations, we have detrended the Calar Alto 2000 and 2003 radial velocity measurements with a sine wave adopting the frequency of the strongest peak in the corresponding periodogram, $3.53 \mathrm{~h}$ and $3.45 \mathrm{~h}$, respectively. The Scargle periodograms of the detrended data (Fig. 11; left panel) contain significant power at frequencies which are consistent with the orbital period derived from the photometry (Sect. 3): $81.26 \pm 0.96 \mathrm{~min}$ (Calar Alto 2000), $81.08 \pm 0.18 \mathrm{~min}$ (Calar Alto 2003), and $81.08 \pm 0.04 \mathrm{~min}$ (combining both sets). Whereas the signal of the orbital period completely dominates the periodogram of the 2000 data, significant residual power is left near $3.45 \mathrm{~h}$ in 


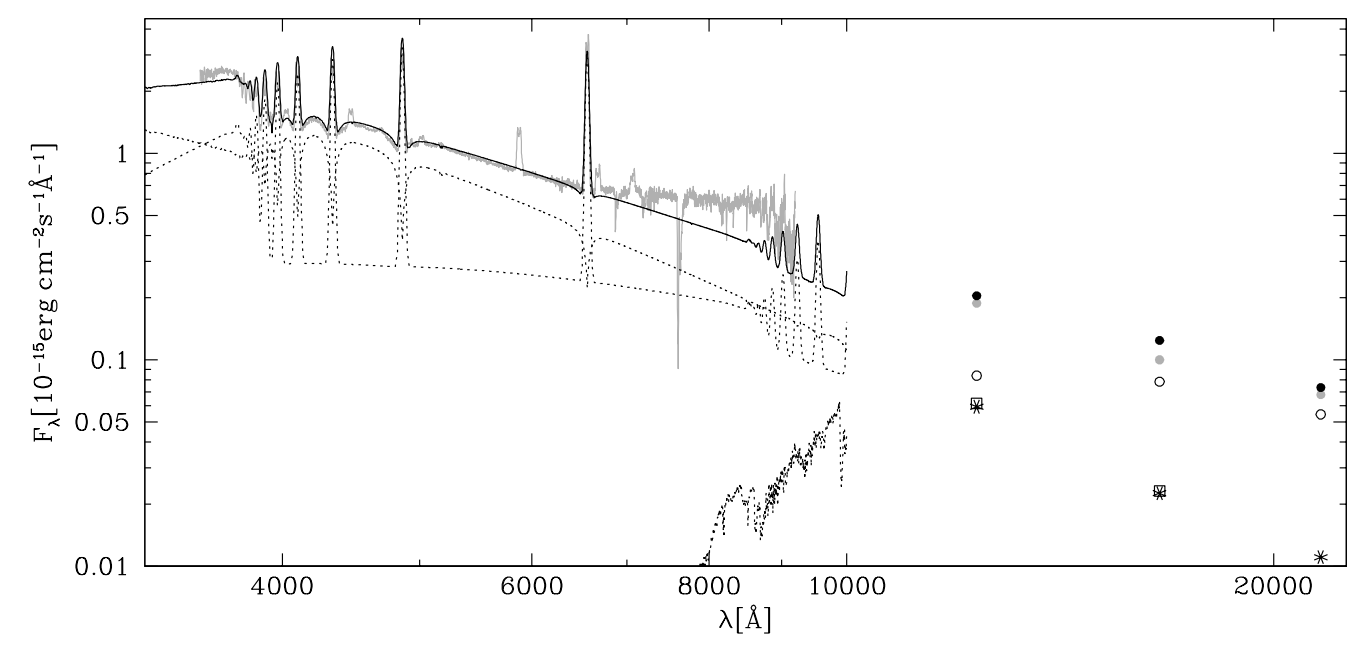

Fig. 12. Three component model to the optical-IR spectral energy distribution of HS 2331. Plotted in gray are the observed average spectrum from Fig. 4 along with the 2MASS $J, H$, and $K$ fluxes (filled circles). Plotted in dotted lines are the white dwarf model (open squares denote the IR fluxes), the isothermal slab model representing the optically thin disc emission (stars denote the IR fluxes), and the spectral contribution of a L2 donor star (open circles denote the IR fluxes). The sum of the three components is shown as black solid line and as black filled circles.

the case of the 2003 data. The main difference between the two data sets is the extent of the observations. While the 2000 data were obtained during two consecutive nights, the 2003 data was obtained during three nights spanning a total of six nights. As the dominant radial velocity variation drifts on times scales of a few days, detrending with a sine with a fixed period will become increasingly imperfect with increasing length of the observing run. Hence, the residual low-frequency power in the case of the 2003 data reflects the imperfection of the applied detrending.

Folding the detrended radial velocities over the orbital period (i.e. $81.08 \mathrm{~min}$ ) results in a sinusoidal radial velocity curve (Fig. 11; right panel), which is smoother for the 2000 data than for the 2003 data, probably again a result of the cleaner detrending possible for these observations. The consistent detection of the 81.08 min period both, in the photometry and in the spectral radial velocity variations lends further support for our conclusion that this is indeed the orbital period of HS 2331.

A sine fit to the folded detrended radial velocities of the Calar Alto 2000 data (top right panel of Fig. 11) results in an amplitude of $\simeq 32 \mathrm{~km} \mathrm{~s}^{-1}$, a systemic velocity of $\sim 1 \mathrm{~km} \mathrm{~s}^{-1}$, and a phase offset with respect to mid eclipse of $\sim 13^{\circ}$. The amplitude, interpreted as radial velocity of the white dwarf $K_{1}$ as it is commonly done, is entirely consistent with what is observed in short-period CVs (e.g. Thorstensen \& Fenton 2003). The phase offset with respect to the expected motion of the white dwarf is most likely related to the fact that the eclipse is caused by an obscuration of the hot spot in the accretion disc, and not of the white dwarf itself. In addition, even in "well-behaved" CVs the broad wings of the emission lines do not necessarily exactly trace orbit of the white dwarf, most likely because of slight asymmetries in the disc.

\subsection{A model for the spectral energy distribution}

We have combined our optical data with the 2MASS $J, H$, and $K$ magnitudes of HS 2331 (Fig. 12), and modeled the optical/IR spectral energy distribution (SED) with a three-component model comprising: (1) a synthetic white dwarf spectrum to account for the observed broad Balmer absorption lines. A grid of model spectra covering $T_{\text {eff }}=8000-15000 \mathrm{~K}$ was calculated with TLUSTY and SYNSPEC (Hubeny 1988; Hubeny \& Lanz 1995). We fixed the surface gravity to $\log g=8.0$, corresponding to $M_{\mathrm{wd}} \simeq 0.6 M_{\odot}$, and the photospheric abundances to 0.1 of their solar values. (2) The emission of an isothermal and isobaric slab with a finite depth to account for the observed Balmer emission lines and any associated continuum. This model is described in detail in Gänsicke et al. $(1997,1999)$. In brief, the free parameters for the generation of this component were the temperature of slab and the column density of the slab along the line of sight. The temperature accounts for the ionisation/excitation of the lines and the optically thick blackbody envelope, and the column density determines the ratio in optical depth between continuum and lines, i.e. the equivalent width of the lines. (3) A late-type spectral template to account for the emission of the secondary star in HS 2331 . The library of templates covered spectral types M 0.5 to M 9 from Beuermann et al. (1998) and L0 to L8 from Kirkpatrick et al. (1999, 2000). The L-dwarf type stars were normalized to surface fluxes using the radii and distances given by Dahn et al. (2002), the M-dwarf templates provided by Beuermann were already normalized in this way.

We started by approximately modeling the observed broad Balmer absorption lines with a white dwarf model, which suggested $T_{\text {eff }} \sim 10000 \mathrm{~K}$ and a white dwarf flux contribution of $\sim 80 \%$ at $5000 \AA$ (Fig. 12). The next step was to extrapolate the white dwarf model established in this way into the FUV wavelength range covered by STIS (Fig. 13). Interpreting the sudden flux up-turn at wavelengths $\lambda \gtrsim 1650 \AA$ as photospheric emission from the white dwarf we refine the white dwarf temperature to $T_{\text {eff }} \simeq 10500 \pm 250 \mathrm{~K}$. The red end of the STIS range is very sensitive to the white dwarf temperature because of the strong temperature dependence of the quasimolecular $\mathrm{H}_{2}$ absorption at $1600 \AA$. The flux scaling factor 


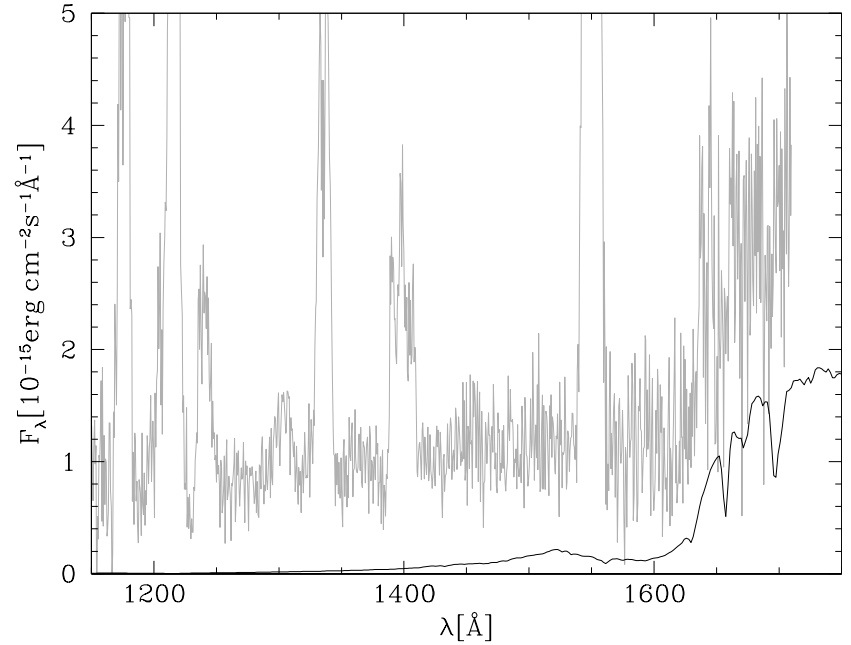

Fig. 13. Gray line: the STIS FUV spectrum of HS 2331 from Fig. 5. Black line: the white dwarf model from Fig. 12 extended into the STIS range. The spectrum of a $\sim 10500 \mathrm{~K}$ white dwarf reproduces well the sudden increase of flux observed longwards of $1600 \AA$.

of our white dwarf model implies a distance of $\sim 90 \mathrm{pc}$. The systematic error in this distance estimate depends primarily on the assumed white dwarf mass, assuming a mass in the range $0.4-0.9 M_{\odot}$ results in $d=90 \pm 15 \mathrm{pc}$. A low distance to HS 2331 is supported by the large proper motion of the star, $\mu \simeq 0.14^{\prime \prime} / \mathrm{yr}^{-1}$, which has been derived from the comparison of the DSS1 and DSS2 images of the system. An isothermal/isobaric slab with a temperature of $T=6500 \mathrm{~K}$ and a surface density of $\Sigma=1.8 \times 10^{-2} \mathrm{~g} \mathrm{~cm}^{-2}$ accounts well for the observed Balmer emission and the Balmer jump, and contributes $\sim 20 \%$ of the flux at $5000 \AA$. Assuming $d=90 \mathrm{pc}$ the area of this simple "disc" component is comparable to the size of the white dwarf Roche lobe. The red end of our optical spectrum shows no evidence for the $\mathrm{TiO}$ absorption bands that are observed in CVs where the late-type donor star significantly contributes to the red/near-IR spectrum. In the last step of our SED modeling we add the spectral contribution of the secondary, assuming $R_{2}=8 \times 10^{9} \mathrm{~cm}$ (corresponding to $P_{\text {orb }}=81.08 \mathrm{~min}$ and $q=M_{\mathrm{wd}} / M_{\mathrm{sec}}=9$; see Sect. 5.1). The flux limit imposed by the 2MASS $J, H$, and $K$ measurements constrain the spectral type of the secondary to be $\sim \mathrm{L} 2$ or later (Fig. 12).

Whereas our three-component model of the FUV-to-IR SED of HS 2331 is overall rather satisfying, two regions of excess flux are observed: the FUV continuum below $\sim 1600 \AA$ significantly exceeds the predicted white dwarf flux (the simple "disc" model and the secondary contribute no flux in the FUV), and the optical continuum in the range $\sim 7000-9000 \AA$ exceeds the flux of the sum of all three components. We believe that the most likely explanation for this excess flux is that our model for the disc emission is too naive - we just assume a single temperature/density pure hydrogen slab. The FUV emission lines clearly indicate that the disc contains hotter regions, which will contribute to some extent to the FUV continuum. The excess flux observed at the red end of our optical spectrum may be related to the Paschen continuum of a somewhat colder part of the disc. It is likely that a multi-temperature/density model of the quiescent disc could account for the observed excess flux, however, we feel that the number of additional free parameters does not warrant this exercise.

\section{Discussion}

Before discussing the nature of the system, we briefly summarise the results of our analysis.

1. HS 2331 has been identified as a CV because of its emission lines detected in the HQS.

2. Coherent low-amplitude eclipses detected over four years unequivocally determine the orbital period to be $P_{\text {orb }}=$ $81.08 \mathrm{~min}$. The mean light curve of HS 2331 bears extreme similarity to that of WZ Sge.

3. The optical and FUV spectrum of HS 2331 is dominated by the photospheric emission of a white dwarf with $T_{\text {eff }} \simeq$ $10500 \mathrm{~K}$.

4. The power spectrum of our optical photometry contains a number of significant signals in the range 5-6 min, which we interpret as non-radial ZZ Ceti pulsations.

5. Additional signals are found at periods of $1.12 \mathrm{~min}$ and $0.56 \mathrm{~min}$, which we tentatively identify as the spin period of the white dwarf and its first harmonic.

6. The distance of the system is $d \simeq 90 \pm 15 \mathrm{pc}$, derived from modelling the emission of the white dwarf. This low distance is consistent with the high proper motion of the system, $\mu \simeq 0.14^{\prime \prime} / \mathrm{yr}^{-1}$.

7. Combining our optical spectroscopy of HS 2331 with the 2MASS $J, H$, and $K$ magnitudes of the system, and adopting $d=90 \mathrm{pc}$ and a mass ratio of $q=M_{\mathrm{wd}} / M_{\mathrm{sec}}=9$ (see below, Sect. 5.1), we find that the donor star must be of extreme late spectral type, L2 or later.

8. Our extensive optical photometry consistently contains a strong signal at a period somewhat longer than $P_{\text {orb }}$. Selecting the alias of that pattern which is closest to $P_{\text {orb }}$ gives $83.38 \mathrm{~min}$, which is $2.8 \%$ longer than the orbital period.

9. Our spectroscopic data set reveals consistently the presence of large-amplitude radial velocity variation with a period of $\sim 3.5 \mathrm{~h}$. However, this radial velocity modulation is not strictly coherent, but appears to drift in either period or phase on a time scale of days.

10. After detrending the radial velocity measurements with a $3.5 \mathrm{~h}$ sinusoid, a radial velocity variation with a period of $81.08 \pm 0.04$, i.e. $P_{\text {orb }}$, is detected with an amplitude of $\simeq 32 \mathrm{~km} \mathrm{~s}^{-1}$.

\subsection{The evolutionary state of HS $2331+3905$}

A number of arguments suggest that HS 2331 is a CV in a late stage of its evolution. Its orbital period is close to the observed period minimum of CVs. A very low mass transfer rate is indicated by the low temperature of the white dwarf, which is a measure of the secular mean accretion rate (Townsley \& Bildsten 2003). This is consistent with the absence of observed outbursts. All our detailed photometric and spectroscopic 
campaigns carried out since September 2000 have found HS 2331 near $V \simeq 16.5$. The CCD-equipped $0.37 \mathrm{~m}$ Rigel Telescope of the University of Iowa has been used to monitor HS 2331 on 16 nights in October to December 2003 and on 15 nights in May/June 2004, finding the system at an instrumental magnitude of $16.4 \pm 0.1$. Additional visual monitoring of HS 2331 has been carried out during 250 nights since March 2003 using a 8 " Schmidt-Cassegrain telescope, with no gaps in the coverage being longer than one week. On no occasion has the star been detected, with a limiting magnitude of $\sim 13.5 \pm 0.5$. In many aspects, HS 2331 resembles the well-studied short-period dwarf nova WZ Sge, which shows extremely bright superoutbursts every $2-3$ decades. If this analogy were correct, we would predict an outburst magnitude for HS 2331 of $V \simeq 9-10$.

Yet another indication of HS 2331 being an old evolved CV is the very late spectral type of the secondary star. Assuming a white dwarf mass of $0.6 M_{\odot}$ and a secondary mass of $0.07 M_{\odot}$ (as it may be realistic for $M_{\mathrm{sec}}$ near the minimum period), i.e. $q \simeq 9$, and a distance of $90 \mathrm{pc}$, our model for the SED of HS 2331 suggests a spectral type later than L2 (Sect. 4.2), which makes this system one of the best candidates for a brown dwarf donor. Detailed infrared spectroscopy will be required to test this hypothesis.

Using the same stellar mass assumption and an inclination of $\simeq 75^{\circ}$, based on the detection of grazing eclipses in the optical light curve, the radial velocity variation of the white dwarf is expected to be $K_{1} \simeq 36 \mathrm{~km} \mathrm{~s}^{-1}$, which is consistent with the amplitude of the $81.08 \mathrm{~min}$ radial velocity variation detected in the spectroscopy, after detrending the data for the dominant $3.5 \mathrm{~h}$ modulation (Sect. 4.1.2).

\subsection{A permanent superhumper?}

From our four years of photometric data, HS 2331 appears to be a permanent superhumper with a likely superhump period of $P_{\mathrm{sh}}=83.38 \mathrm{~min}$, i.e. a period excess $\left(P_{\mathrm{sh}}-P_{\mathrm{orb}}\right) / P_{\mathrm{orb}}=2.8 \%$. This finding is exceptional, as superhumps are normally detected during superoutbursts of SU UMa type dwarf novae or in some nova-like variables during their high states, i.e. always during phases of high mass transfer in the accretion disc. Superhumps are thought to be related to the precession of tidally unstable eccentric accretion discs in close binaries with extreme mass ratios (e.g. Osaki 1985; Murray 1998).

If our interpretation of the photometric signal is correct, and indeed $P_{\mathrm{sh}}=83.38 \mathrm{~min}$, the implied period excess of $2.8 \%$ is not particularly small (Patterson 2001), somewhat arguing against an extreme mass ratio in HS 2331. However, considering the unique nature of HS 2331, it is not clear if Patterson's relation can be directly adopted for this system.

The strangest and most disconcerting observational fact about HS 2331 is without doubt the detection of a dominant radial velocity variability with a period of $3.5 \mathrm{~h}$, which does not correspond to the orbital motion of the system. This periodicity resulted from measuring changes in the wings of the Balmer emission lines and therefore reflects variability in the inner disc region of the systems, however we have at present no explanation for the physical origin/significance of its $3.5 \mathrm{~h}$ period. Again, in a very speculative approach, we suggest that the period (or phase) drift of this radial velocity variation may be related to the presence of permanent superhumps. The precession period of the accretion disc implied by the orbital period and the superhump period is $\sim 2 \mathrm{~d}$, which is comparable to the time scale on which the $3.5 \mathrm{~h}$ radial velocity variation varies. An intensive spectroscopic campaign covering several of the putative precession cycles could test this hypothesis.

Another important conclusion from the radial velocity analysis is that care should be taken when implicitely assuming that the dominant radial velocity variation detected in cataclysmic variables (and related objects) reflects the orbital period. Whereas this is contrary to the customary approach for the past decades, the results obtained for HS 2331 are a clear warning that this custom could occasionally lead to wrong interpretations.

\subsection{The brightest CV white dwarf pulsator}

Until recently, only a single CV containing a white dwarf pulsator has been discovered: GW Lib (van Zyl et al. 2000, 2004). GW Lib is only partially understood, due to the lack of a proper mode identification of the observed pulsation frequencies, and more severely, due to the circumstance that the temperature of its white dwarf, $T_{\text {eff }}=14700 \mathrm{~K}$, is actually significantly above the blue end of the ZZCeti instability strip (Szkody et al. 2002). Three additional CVs containing pulsating accreting white dwarfs have been discovered in the SDSS: SDSS J013132.39-090122.3, SDSS J161033.64010223.3, and SDSS J220553.98+115553.7 (Warner \& Woudt 2003; Woudt \& Warner 2004).

We have shown that HS 2331 exhibits the photometric behaviour typical of ZZCeti pulsators, showing a multiperiodic and complex power spectrum in the range $\sim 60 \mathrm{~s}$ to $\sim 300 \mathrm{~s}$. With $V \simeq 16.5$, HS 2331 is by far the brightest $\mathrm{CV}$ white dwarf pulsator, making it the prime target for detailed followup studies. The white dwarf temperature implied by the SED of HS $2331\left(T_{\mathrm{eff}} \simeq 10500 \mathrm{~K}\right.$, assuming $M_{\mathrm{wd}} \simeq 0.6 M_{\odot}$, Sect. 4.2) is just slightly below the red end of the ZZ Ceti instability strip for single white dwarfs (e.g. Bergeron et al. 2004). The ultra-short periods detected in HS 2331, with periods of $1.12 \mathrm{~min}$ and $0.56 \mathrm{~min}$ which are too small for conventional $g$-mode pulsations, remains a puzzle. One possible solution is to assume that $1.12 \mathrm{~min}$ is the white dwarf spin period, and $0.56 \mathrm{~min}$ its first harmonic. Polarimetry and X-ray detection of the 1.12 min period would be the ultimately proof of this hypothesis.

A more detailed understanding of the white dwarf pulsations in CVs is just at its beginning, as the first models for the driving mechanism are being developed (Townsley et al. 2004), and a larger sample of these objects is discovered. Future potential asteroseismological applications in CV white dwarfs include measuring the white dwarf masses, as well as studying the accreted envelope. 


\section{Conclusion}

We have discovered a new short orbital period CV, HS 2331, which displays an overwhelming wealth of observable phenomena, and we encourage detailed follow-up studies over all wavelength ranges and time scales to exploit the opportunities that this system offers for an improved understanding of CV evolution and accretion onto white dwarfs.

Acknowledgements. We thank the referee, Patrick Woudt, for a prompt and helpful report. S.A.B. acknowledges support from NASA through grants GO-9357 and GO-9724 from the Space Telescope Science Institute, which is operated by AURA, Inc., under NASA contract NAS5-26555. B.T.G. was supported by a PPARC Advanced Fellowship, The HQS was supported by the Deutsche Forschungsgemeinschaft through grants Re 353/11 and Re 353/22. We are very grateful to Klaus Beuermann and Davy Kirkpatrick for giving us access to their spectral templates for M- and L-dwarfs. We would also like to thank Pablo Rodriguez-Gil, Matthias Schreiber, Klaus Beuermann, Luisa Morales, Dean Townsley, Lars Bildsten and Phil Arras for helpful discussions and to Spiridon Kitsionas for a careful reading of the manuscript. P. Schmeer is very grateful to Robert Mutel and his students for scheduling some of the CCD monitoring of HS 2331. We thank the director of the Calar Alto observatory for the generous allocation of discretionary time for this project.

\section{References}

Araujo-Betancor, S., Gänsicke, B. T., Hagen, H.-J., Rodriguez-Gil, P., \& Engels, D. 2003, A\&A, 406, 213

Bergeron, P., Fontaine, G., Billères, M., Boudreault, S., \& Green, E. M. 2004, ApJ, 600, 404

Beuermann, K., Baraffe, I., Kolb, U., \& Weichhold, M. 1998, A\&A, 339,518

Beuermann, K., Wheatley, P., Ramsay, G., Euchner, F., \& Gänsicke, B. T. 2000, A\&A, 354, L49

Castanheira, B. G., Kepler, S. O., Moskalik, P., et al. 2004, A\&A, 413, 623

Clemens, J. C. 1993, Baltic Astron., 2, 407

Dahn, C. C., Harris, H. C., Vrba, F. J., et al. 2002, AJ, 124, 1170

Dhillon, V., Marsh, T. R., Kelly, J., et al. 2002, in The Physics of Cataclysmic Variables and Related Objects, ed. B. T. Gänsicke,

K. Beuermann, \& K. Reinsch, ASP Conf. Ser., 261, 672

Filippenko, A., \& Greenstein, J. L. 1984, PASP, 96, 530

Gänsicke, B. T., Araujo-Betancor, S., Hagen, H.-J., et al. 2004, A\&A, 418,265

Gänsicke, B. T., Szkody, P., de Martino, D., et al. 2003, ApJ, 594, 443

Gänsicke, B. T., Beuermann, K., de Martino, D., \& Thomas, H.-C. 2000, A\&A, 354, 605
Gänsicke, B. T., Beuermann, K., \& Thomas, H. C. 1997, MNRAS, 289, 388

Gänsicke, B. T., Hagen, H. J., \& Engels, D. 2002, in The Physics of Cataclysmic Variables and Related Objects, ed. B. T. Gänsicke, K. Beuermann, \& K. Reinsch, ASP Conf. Ser., 261, 190

Gänsicke, B. T., Sion, E. M., Beuermann, K., et al. 1999, A\&A, 347, 178

Hagen, H. J., Groote, D., Engels, D., \& Reimers, D. 1995, A\&AS, 111,195

Horne, K. 1986, PASP, 98, 609

Hubeny, I. 1988, Comput. Phys. Comm., 52, 103

Hubeny, I., \& Lanz, T. 1995, ApJ, 439, 875

Kepler, S. O., Nather, R. E., Winget, D. E., et al. 2003, A\&A, 401, 639

King, A. R. 1988, QJRAS, 29, 1

King, A. R., \& Schenker, K. 2002, in The Physics of Cataclysmic Variables and Related Objects, ed. B. T. Gänsicke, K. Beuermann, \& K. Reinsch, ASP Conf. Ser., 261, 233

Kirkpatrick, J. D., Reid, I. N., Liebert, J., et al. 1999, ApJ, 519, 802

Kirkpatrick, J. D., Reid, I. N., Liebert, J., et al. 2000, AJ, 120, 447

Kolb, U. 1993, A\&A, 271, 149

Littlefair, S. P., Dhillon, V. S., \& Martín, E. L. 2003, MNRAS, 340, 264

Mauche, C. W., Lee, Y. P., \& Kallman, T. R. 1997, ApJ, 477, 832

Murray, J. R. 1998, MNRAS, 297, 323

Oke, J. B. 1990, AJ, 99, 1621

Osaki, Y. 1985, A\&A, 144, 369

Patterson, J. 1998, PASP, 110, 1132

Patterson, J. 2001, PASP, 113, 736

Patterson, J., Richman, H., Kemp, J., \& Mukai, K. 1998, PASP, 110, 403

Rodríguez-Gil, P., Gänsicke, B. T., Araujo-Betancor, S., \& Casares, J. 2004, MNRAS, 349, 367

Scargle, J. D. 1982, ApJ, 263, 835

Schneider, D. P., \& Young, P. 1980, ApJ, 238, 946

Shafter, A. W. 1983, ApJ, 267, 222

Szkody, P., Gänsicke, B. T., Howell, S. B., \& Sion, E. M. 2002, ApJ, 575, L79

Thorstensen, J. R., \& Fenton, W. H. 2003, PASP, 115, 37

Thorstensen, J. R., Taylor, C. J., \& Kemp, J. 1998, PASP, 110, 1405

Townsley, D. M., Arras, P., \& Bildsten, L. 2004, ApJ, 608, L105

Townsley, D. M., \& Bildsten, L. 2003, ApJ, 596, L227

van Zyl, L., Warner, B., O’Donoghue, D., et al. 2004, MNRAS, 350, 307

van Zyl, L., Warner, B., O’Donoghue, D., et al. 2000, Baltic Astron., 9,231

Warner, B., \& Woudt, P. 2003, in Variable Stars in the Local Group, ed. D. Kurtz, \& K. Pollar, in press [arXiv: astro-ph/0310072]

Woudt, P. A., \& Warner, B. 2004, MNRAS, 348, 599

Woudt, P. A., Warner, B., \& Pretorius, M. L. 2004, MNRAS, 351, 1015 